\title{
Educational aspects of cosmology Global Cosmology Teachers Academy
}

\author{
George F. Smoot ${ }^{1,2,3}$ \\ ${ }^{1}$ Lawrence Berkeley National Lab, \\ 1 Cyclotron Road, Astrophysics Group, MS 50-5005, Berkeley, CA 94720, USA \\ ${ }^{2}$ Institute for the Early Universe, \\ Ewha Womans University, 11-1 Daehyun-Dong Seodaemun-Gu, Seoul 120-750, Korea \\ ${ }^{3}$ Laboratoire Astroparticules et Cosmologie, Université Paris Diderot-Paris 7, \\ Bâtiment Condorcet, Case 7020, 75205 Paris Cedex 13, France
}

email: GFSmoot@lbl.gov

\begin{abstract}
This IAU-UNESCO Symposium 260 'The role of Astronomy in Society and Culture' celebrates the 400th anniversary of the beginning of modern astronomy marked by when Galileo turned a telescope to the sky making so many astounding discoveries that changed our perceived position in the solar system. This talk celebrates that cosmology has entered this era in an even deeper and profound discoveries that have changed our perception of the universe and our place in it. As such cosmology is a great interest and educational tool for capturing the attention of the young and broadening their perspective. The first portion of the talk outlines and reveals some of these observations and the second with examples and vision of how this can provide an encompassing storyline for STEM learning. This proceedings also emphasizes the key role that partnerships including the private sector are valuable, critical, and rewarding part of the effort.
\end{abstract}

Keywords. Science education, STEM learning

\section{Introduction}

This symposium kicks off the Year of Astronomy and it is appropriate to quote Catherine Cesarsky : "By the end of the year, we would like everyone on earth to have spent at least a short while with their attention turned to the sky. Or at least to have read something on the most recent discoveries or reflected on our position in the universe" $\dagger$. In my view seeing the sky makes a deep impression and makes one think about larger issues and reflect and want to know more about the universe and the much larger world out there.

This is why cosmology is so key in STEM education and human culture and science. $21^{\text {st }}$ Century cosmology is:

- The universal story of origins that humans have sought through the ages

- A culmination of science, engineering, mathematics and technology

- An encompassing story line for STEM learning.

To illustrate the power and pull of cosmology we take a little trip that I simply title: The Dawning of the Universe - How we know about our origins by studying the relics of creation. One merely needs to look a the Hubble (our current best version of Galileo's telescope) ultra-deep field image and to see the thousands of galaxies in this image showing up behind a very few stars from our own galaxy. The overwhelming concept is that we are seeing so many galaxies of $10^{11}$ (100 billion) stars and that this Hubble image

$\dagger$ as interviewed by Pierre Gaillard, Bureau of Public Information, UNESCO. 
is only a tiny fraction of the sky so that if surveyed we would be able to image about 100 billion galaxies in our visible universe. This means that there are in the observable universe about 3 to $7 \times 10^{22}$ stars. In turn that implies that there is of order about $10^{22}$ (10 septillion) planetary systems. This is only what we can see, we anticipate that surely the universe is much, much larger than what we can see. This raises many, many questions about our place in the universe and about the universe including where did it come from and why are there so very many galaxies.

This simple short trip to the universe is compelling indication of why cosmology has its pull and impact on us all and stimulates the young to learn more.

\section{Story Tools}

(a) Looking out into space is also looking back in time because of the light travel time, and because the universe is so large (and empty).

(b) Cosmic Spheres of time

(c) The relic radiation from the beginning, the Big Bang

(d) The grand sweep of history our world line to the here and now

(e) Giant surveys of millions of galaxies

$(f)$ Models and Simulations - more and bigger simulations

\section{Global Cosmology Teachers Academy Concepts}

Why Global? Knowledge of the history and structure of the universe and how we know about it provides:

- A common heritage of our place in space and time

- A foundation for teaching and learning supporting STEM literacy

- A educational context for inspiration and innovation for future scientists and engineers

- It is truly a story that is the common origin of mankind.

Education - What is needed?

- A commitment and support for literacy for all.

- Qualified and motivated teachers are needed around the world for basic literacy (United Nations Literacy Decade 2003-2012)

- Scientific and Technological Literacy (TIMSS reports)

- Coherent curriculum for elementary, middle, and secondary

- Qualified teachers excited about the story they have to tell

- $21^{\text {st }}$ Century STEM education for innovative scientists and engineers to solve global problems is also vital for economic growth and cultural improvement

\section{1st Century STEM Education}

The context is changing from:

- Limited to ubiquitous access to knowledge and resources

- Restricted to unrestricted communications

- Access from simple to sophisticated computational tools and real world data

- Learning facts and concepts to utilizing and applying knowledge (PISA findings)

- National to international competition and cooperation

- Fully integrated science, technology, engineering, and mathematics K-12 education

This change in context is part of the innovation and transformation of world communications and commerce. These call for a new model of STEM education to handle the current and emerging landscape. We must make the ubiquitous access to knowledge as much a boon to the teachers as it can be to the coming generations of students. By taking 
a global approach we can foster the international cooperation and friendly competition that encourages greater achievements.

\section{The Berkeley Center for Cosmological Physics (BCCP)}

Fully integrated research and education with:

- International collaborations including some of the most audacious and exciting advance projects.

- Involvement of front-line research scientists, post doctoral scholars, graduate students, and undergraduate students in education and outreach.

- Global Teachers Academy to advance 21st century STEM education using cosmology as a unifying story line.

Features of the BCCP Global Teachers Academy

- Staffed by experienced and recognized science educators

- A coherent story line based upon cosmology that facilitates STEM teaching and learning

- A richness of teaching and learning resource materials including equipment for hands-on investigations

- Recognition, renewal, and revitalization of teachers through association with BCCP

- Access to scientists, engineers, post doctoral scholars, graduate and undergraduate students

- Face-to-Face teacher leadership training at the Berkeley Center for Cosmological Physics

- Cyber training and support for teacher communities

- Students interacting with students around the world through the web to share their growing knowledge in the context of their local environment and culture.

Access for teachers and students to:

- Real science, engineering and computational tools and data

- Real scientific and engineering challenges

- Real scientists, engineers, computer scientists, etc.

Support for teachers and students to:

- Collaborate beyond school, state, and national boundaries to the whole world

- Investigate STEM related problems and publish results

- Communicate from the class room with students, teachers, scientists and engineers around the world

Teachers participate in a two week summer workshop with master teachers and scientists from the Berkeley Cosmology Center. Later there is follow up through various channels but most especially via internet and our web support for teachers. Teachers and their students receive support throughout the academic year. This support includes funding for presenting workshop lessons to teacher colleagues, and to meet for a three day conference the following summer to present their lessons and assessment results.

Working in groups with talented high school students, undergraduate students preparing for teaching careers and master teachers experience a technology rich, project based learning environment. Teachers are introduced to cosmology and learn how science, engineering, and math concepts create the foundation for student understanding of the 
structure and evolution of the universe. They are introduced to online resources, support, and tools for teaching. Participants create a professional development plan that includes the teaching and assessment of a project based lesson with their students during the academic year.

Our primary focus is on teachers of middle and high school students. We engage the theme of our place in the universe as a rich story line of universal interest to unite standards for science and math content. This further integrates science, technology, engineering and mathematics teaching and learning.

\subsection{Teachers Recognition, Reward, and Revitalization}

A goal is to assist in new teacher development but also in the recognition, reward, and revitalization of current science and math teachers. This includes

- Updated science content knowledge

- Experience with project based learning

- Confidence in using technology in the classroom

- Methods and tools and for scientific inquiry, engineering design and mathematical modeling

- Professional development plan

- Leadership skills

- Access to scientists and engineers

Schools and Administrators gain through creating teacher leaders for 21st Century teaching and learning. The program is consistent with school site and district priorities for STEM education.

Sponsors by partnering with us gain through

(i) Education outreach opportunities for employees

(ii) Local, regional, national and international recognition

(iii) Future employees with 21st century skill and knowledge.

Sponsors provide teachers with access to a world-class, fully integrated research and education community and provide schools and administrators with leaders for their initiatives to implement and improve STEM teaching and learning. They provided communities with excitement, hope, and encouragement where students have access to quality education, and educators for STEM college majors and careers. Sponsor also provide in-kind support through employee participation, and teacher recognition. This kind of public-private partnership is essential to get innovative education and out reach activities to a large section of teachers and communities.

We provide Sponsorship opportunities in several areas to attract partners and companies interested in work force development:

- Corporate engagement opportunities

- Co-branded opportunities to support teacher fellows (Local, national and international)

- Co-branded opportunities to support summer workshop videos (national distribution and audience)

- Co-branded opportunites to support summer workshop participants (diverse high school students) Technology support

- Local, National, and International presence and collaborations (Asian, Europe, U.S.A., Latin America, Africa)

- Education and Outreach Support for GTA 2010, GTA 2011

The BCCPs education programs depend on volunteers for assistance. The Corporate Volunteer Program offers groups of corporate volunteers the opportunity to participate in the hands-on work for the BCCP GTA. The Corporate Volunteer Program projects 
are designed to be enjoyable and fulfilling, and to give you and your employees a sense of ownership in our dynamic Global Teachers Academy. Projects may include preparation and participation at our annual academy (Berkeley-California, Seoul-South Korea, and Paris-France). Most projects require a full-day commitment to complete. Some of our projects involve the opportunity to donate funds towards the purchase of necessary equipment and supplies for our teachers and students, which allows the BCCP GTA to enhance their resources for participants.

An International Partnership Program enhances and broadens the effort. There are current partnerships with the Paris Center for Cosmological Physics, Global HandsOn Universe, and the Institute for the Early Universe in Seoul, Korea. We are in process of establishing more partners and are working with various science centers. In the long term these partnerships will allow us to reach a large portion of the science and math teachers across the world with sensitivity to each region.

We also connect to our graduate, post doctoral and young research school Essential Cosmology For the Next Generation. BCCP and its international partners launched a second school in 2010 and have been successful in establishing a prestigious, annual event that meets the demands of workforce development, diversity in a new science field, education and the advancement of science. Through global distribution, your company brand and message will be displayed on promotional materials, media and other opportunities that promote these priorities. Essential Cosmology For the Next Generation creates the opportunity to support a diverse group of eminent scientists, researchers, students, and innovators in the modern Physics scene. Essential Cosmology for the Next Generation showcases innovations in training, communications and scientific interaction, utilizing the interdisciplinary approach essential for the new generation of cosmological data and interpretations. With world-class lectures, the latest astrophysical results and strong international collaborations, the conference presents a superb opportunity for significant impact on a diverse group of participants and, through high-quality web casting, to a far larger community at U.S. institutions and abroad. In addition, a public talk presented by Nobel Laureate George Smoot is organized with the local community, officials, and supporters invited to participate and interact with great minds in science.

This combined GTA, BCCP, and Essential Cosmology for the Next Generation provides a fully integrated approach to understanding and communicating cosmology and science. This school provides the interest and talent to populate with excited and front line researchers out to the community and to the GTA.

Existing Elements for Success

Access to frontier science and engineering resources, data and programs:

- NASA

- European Space Agency

- Large Hadron Collider

- Kavli Research Centers

- BCCP, IEU, PCCP

Model programs and teacher networks, e.g.,

- Hands-On Universe and Global Hands-On Universe

- GLOBE program

Educational Tools for building STEM literacy

- Concept mapping and writing

- Mathematical tutorials and modeling 


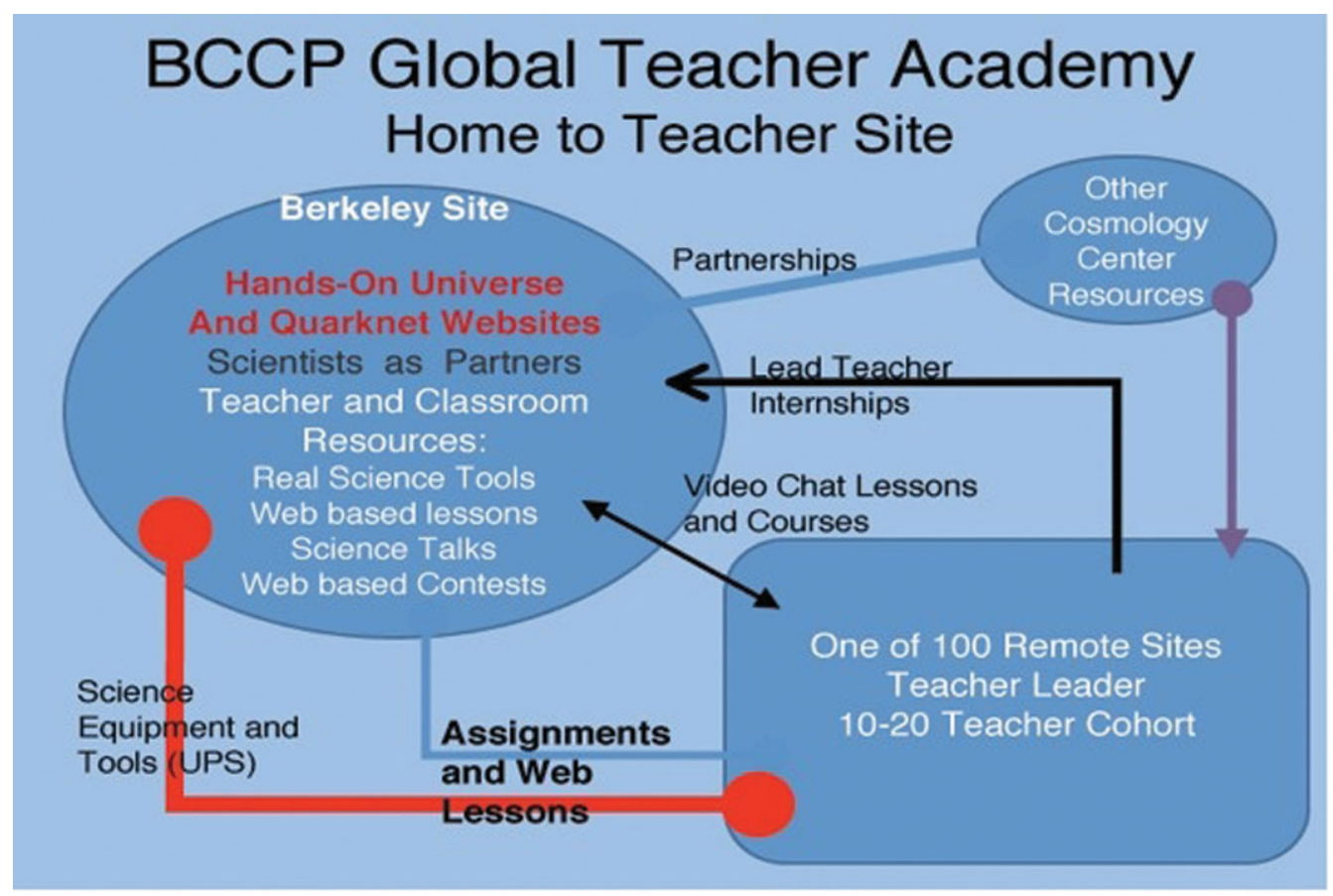

Figure 1. Schematic of Global Teachers' Academy Operations

- Simulations of science concepts and principles

Challenges Teachers Face

- Content Knowledge

- Time

- On-going follow-up support

- Access to educational and technology resources

- Standards, testing and administrative expectations

- Access to science and engineering people and enterprise

\subsection{BCCP Global Teachers Academy}

Designed for building capacity

- Resource for building cosmology threads in curriculum

○ http://UniverseAdventure.orgUniverse Adventure web site

○ http://YouTube.comYouTube Contest

- Follow-up support

- Resources for the classroom

- Cyber-based support for teachers and their students

- Resources for professional conferences

- Support for Teacher Action Research projects

- Teacher leadership recognition and support

Summer Institute for teacher teams

- Primary, Secondary, and Tertiary teacher participants

- Scientists and post doctoral scholars, graduate and undergraduate students

- Model project based learning 


\subsection{BCCP GTA Institute 2009}

Cosmology Threads through the Grade levels

- Our Sun, Earth, Moon system

- Our Solar System

- Our Galaxy

- Our Place in the Universe

Cosmology Frontier Presentations

- CMB, Dark Matter, Dark Energy, Neutrinos, Gravitational Lensing, ..

Hands-On Investigations

- Hands-On Universe astronomy. Hands-On Universe (HOU) is an educational program that enables students to investigate the Universe while applying tools and concepts from science, math, and technology. Global Hands-On Universe joins efforts from all around the world, allowing students and teachers to share their work and experiences.

- Investigations of foundational concepts, principles and theories of Heat, Light, Gravity using Vernier Probeware and Analysis Tools

Teacher Professional Development Proposal

- Design proposal of a cosmology thread for elementary and secondary students

- Teacher professional development planning and introduction to cyber tools for ongoing support and development

\subsection{Rationale and Goals}

Cosmology is the selected field of focus for the academy because it incorporates various fields of science: Physics, Astrophysics, Particle Physics, Theoretical Physics, Astronomy, Earth Science, Math, Engineering, and Technology.

The Global Teachers Academy improves classroom science instruction by training teachers to engage students in a technology rich, project based experience.

We work with sponsors and school administrators, science, technology, engineering and mathematics teachers are selected for leadership development and support. We offer a model for 21st century STEM education that includes:

- Access to frontier science and engineering people, problems, and tools

- Project based learning opportunities

- Utilization of technology for data acquisition, analysis, modeling, information and communication

- Authentic assessment of skills and knowledge

- Science inquiry and engineering design in teaching and learning

- An international partnership program

Teachers and their students have access to and experience learning through exciting partnerships with international organizations to draw upon new ideas, expertise, and resources from around the globe, recognizing that virtually all major initiatives in cosmology require innovative global collaboration.

\section{Vision and conclusion}

Students around the world are learning about their time and place in the universe, its history, structure and the 21st century technologies that makes this knowledge of orgins possible. Teachers have the freedom, resources, and knowledge to enjoy liberating the minds of their students to the 21st century science, technology, engineering, and mathematics. This become a collaborative journey for the students, teachers and their contacts around the world. 